\title{
Solution of the Cauchy problem for a conservation law with a discontinuous flux function
}

\author{
TORE GIMSE ${ }^{1}$ \\ NILS HENRIK RISEBRO ${ }^{1}$ \\ Department of Mathematics \\ University of Oslo
}

\begin{abstract}
We solve the Cauchy problem for a conservation law arising in oil reservoir simulation where the flux function may depend discontinuously on the space variable. To do this we use front tracking as a method of analysis.
\end{abstract}

Introduction. In this paper we study the Cauchy problem for two phase flow through a one dimensional porous medium. Darcy's law together with the equations of mass balance gives

$$
s_{t}+\left\{f_{0}(s)(v-g(x) k(s))\right\}_{x}=0
$$

where $s=s(x, t)$ denotes the saturation of one of the phases, $f_{0}$ is the fractional flow function, $v$ is the total Darcy velocity and $k(s)$ is the relative permeability of the phase not denoted by $s$. The gravitational term $g(x)$ includes the density differences between the phases as well as the absolute permeability of the rock and the angle of dip of the reservoir. This term is therefore not necessarily a continuous function of $x$. Equation (0.1) is an example of a conservation law

$$
\begin{aligned}
& u_{t}+f(u, x)_{x}=0 \\
& u(x, 0)=u_{0}(x)
\end{aligned}
$$

where $u$ may be either a vector or a scalar variable. Such conservation laws do not in general posess continuous solutions, and by a solution of $(0.2)$ we mean a solution in the distributional sense such that for each $\phi \in C_{0}^{1}$

$$
\int_{-\infty}^{\infty} \int_{0}^{\infty}\left(u \phi_{t}+f(u, x) \phi_{x}\right) d t d x+\int_{-\infty}^{\infty} \phi(x, 0) u_{0} d x=0 .
$$

The solution $u$ is then called a weak solution of (0.2). Krushkov proved the existence of a weak solution to $(0.2)$ for a scalar $u$ under the assumption that $\frac{\partial f}{\partial x}$ was bounded[9]. This assumption does not hold for (0.1) since the geology often varies discontinuously in a porous medium.

Here we are interested in the initial value problem for $(0.1)$ and we prove the following theorem:

\footnotetext{
${ }^{1}$ Supported by the Royal Norwegian Council for Technical and Industrial Research
} 
THEOREM. If $g(x)$ has bounded total variation, (0.1) posesses a weak solution $s(x, t)$ for arbitrary initial data $s_{0}(x)$ of bounded total variation.

This theorem is proved through construction of a sequence of approximate solutions. These solutions are constructed by the method of front tracking introduced by Dafermos[1] and developed by Holden et al.[4]. This front tracking method is based on the solution of the Riemann problem for (0.1) which was studied by the authors in [3] and here we give a brief review of its solution. The solution of the Riemann problem for $(0.1)$ is similar to the solution of the Riemann problem for the oil-polymer system studied by Isaacson[6]. This similarity is sufficient for us to use some of the ideas developed by Temple[11] for the oilpolymer system, most notably the construction of a mapping $\Psi$ from $(s, g)$ to $(z, g)$ such that the total variation of the approximate solutions remains bounded in $(z, g)$. We will define a functional $F=F\left(u_{\delta}\right)$ where $u_{\delta}$ is our approximate solution generated by the front tracking scheme. Then $F$ is shown to be nonincreasing in time and this enables us to show that the sequence of approximations is well defined in the sense that each approximate solution can be defined at any time. Furthermore we show that each approximate solution is constant on a finite number of polygons in $x-t$ space. Via a standard compactness argument we can now show that a subsequence of the approximate solutions converges. The approximate solutions are constructed in such a way that they are weak solutions of equations which are close to (0.1). This makes it straightforward to prove that the limit is a weak solution.

In the first section of this paper we give some of the "physics" of the problem which leads to $(0.1)$. In section two we review the solution of the Riemann problem. In section three we present the front tracking scheme and introduce the mapping $\Psi$ and the functional $F$. We then show that $F$ is nonincreasing and that this implies that the approximations are well defined. In section four we prove that a subsequence of the approximate solutions converges towards a weak solution. Finally we make a remark on the applicability of this method of analysis to the oil-polymer system.

Physical motivation. We want to study two-phase flow in porous media, assuming for each phase Darcy's law:

$$
v=-\lambda(\nabla P-\rho G),
$$

where $v$ is the Darcy speed, $\lambda$ is the mobility, $P$ the phase pressure, $\rho$ is the density, and $G$ a gravitational term. Combining Darcys law with the source-free equation of mass conservation for each phase

$$
\rho_{t}+\nabla(v \rho)=0
$$

we find (for a more detailed treatment of these equations see [10]):

$$
\alpha\left(\phi \rho_{w} s_{w}\right)_{t}+\left(\alpha \rho_{w} F_{w}\right)_{x}=0
$$

which is the one-dimensional saturation equation, ignoring capillary effects (diffusion). Here $\alpha$ is the one dimensional crossectional area, $\phi$ is the rock porosity, $\rho_{w}$ is the density of water, and $s_{w}$ is the saturation of the water at position $x$ at time $t$. Lower indices $x$ and $t$ indicate derivatives with respect to space and time respectively. $F_{w}$ is the flow function of water:

$$
F_{w}=f_{w}\left(v-K \lambda_{o}\left(\rho_{w}-\rho_{o}\right) g\right)
$$


where $f_{w}$ is the fractional flow function of water, $f_{w}=\frac{\lambda_{w}}{\lambda_{w}+\lambda_{o}}, \lambda_{w}$ and $\lambda_{o}$ being the phase mobilities of water and oil, $v$ is the total Darcy velocity, $K$ is the absolute rock permeability, and $g$ is the component of gravity along the reservoir. Even if $\alpha, \phi$, and $\rho$ are constants, so that (1.1) simplifies to read:

$$
\left(s_{w}\right)_{t}+\frac{1}{\phi}\left(F_{w}\right)_{x}=0
$$

$F_{w}$ may be a function of position as well as saturation, $F_{w}=F_{w}\left(s_{w}, x\right)$. Heterogenities like a varying reservoir angle (and thereby changing $g$ ), or changes in the rock permeability, $K$, along the reservoir, may both affect the flow function. This positional dependence of $F_{w}$ may be smooth, when the parameters vary continuously along the reservoir, or discontinuous. The latter is probably very important and perhaps more common, since the rock is usually layered to some extent throughout the reservoir. Between such layers, introducing abrupt changes in rock permeability, $K=K(x)$ should be modelled discontinuously.

In general, the phase mobility curves $\lambda=\lambda\left(s_{w}\right)$ are assumed to be convex functions, typically shaped as indicated in figure 1.1. This gives an s-shaped, increasing fractional flow function $f_{w}=f_{w}\left(s_{w}\right)$, as shown in figure 1.2. In general, with increasing gravity or permeability, $f$ decreases, so that two different flow functions typically look like in figure 1.3. We will be interested in the Cauchy problem for (1.3).

The Riemann problem. We let $s$ denote the saturation variable, introduce a variable $g=g(x)$ representing the geology, and let $u=(s, g)$, so that (1.3) may be written as a so-called triangular system ([2] [5]):

$$
\begin{aligned}
& u_{t}+f(u)_{x}=0, \\
& u(x, 0)=u_{0}(x) .
\end{aligned}
$$

Here $f(u)=(h(s, g), 0)$ with

$$
h(s, g)=f_{0}(s)(1-g k(s)),
$$

$f_{0}=f_{0}(s)$ being a Lipschitz continuous, increasing function with one point of inflection (s-shaped) with $f_{0}(0)=0$ and $f_{0}(1)=1$ as in figure 1.2. The relative permeability $k(s)$ is usually assumed to be a decreasing, convex function of the saturation such that $k(0)=1$ and $k(1)=0$, cf. figure 1.1. Note that this implies that $h(1, g)=1$ for all $g$. Also each $h(\cdot, g)$ is Lipschitz continuous and has (possibly) one minimum and two points of inflection within the interval of definition, and finally $\frac{\partial h}{\partial g} \leq 0$, cf. figure 1.3. The Riemann problem for (1.3) or (2.1), which is the initial value problem with initial constant states, denoted by $u_{L}=\left(s_{l}, g_{l}\right)$ and $u_{R}=\left(s_{r}, g_{r}\right)$, separated by a single geological discontinuity, has been studied by the authors in [3], where existence and uniqueness results are proved. Here we will need to know the solution of Riemann problems, so before proceeding with a more general treatment of (2.1), we will briefly summarize the main results of [3].

The one dimensional Riemann problem for (2.1) may be written in the form

$$
\begin{array}{ll}
s_{t}+f_{l}(s)_{x}=0 & \text { for } x<0 \\
s_{t}+f_{r}(s)_{x}=0 & \text { for } x>0
\end{array}
$$


with initial data

$$
s(x, 0)= \begin{cases}s_{l}, & \text { if } x<0 \\ s_{r}, & \text { if } x>0\end{cases}
$$

In the above notation, $f_{l}(s)=f_{0}(s)\left(1-g_{l} k(s)\right)$ and $f_{r}(s)=f_{0}(s)\left(1-g_{r} k(s)\right)$. Thus, to the left of the origin the flow function is $f_{l}$ and to the right $f_{r}$. The saturation variable $s$ is in the range $0 \leq s \leq 1$. We define $s_{-}$and $s_{+}$to be the limits of the solution $s(x, t)$ as $x$ approaches zero from below and above respectively. Note that if the solution to (2.2) is unique, these values are independent of $t$. The Hugoniot relation at $x=0$ gives

$$
f_{l}\left(s_{-}\right)=f_{r}\left(s_{+}\right)
$$

The procedure for determining possible values for $s_{-}$and $s_{+}$is explained in full detail in [3], where it is proved that two such points always exist, and by introducing an additional entropy condition for the shock at $x=0, s_{-}$and $s_{+}$are uniquely determined. This entropy condition says that the jump $\left|s_{-}-s_{+}\right|$at $x=0$ should be the smallest possible jump here satisfying (2.3). This minimal jump condition is proved to be equivalent to the viscous profile entropy condition for an enlarged system of equations, in some extent equivalent to (2.2). The reader is refered to [3] for further details. We will now turn our attention to the two different waves involved in the solution of (2.1). First, $s$-waves are defined to be waves of constant $g$. Thus, in $(s, g)$ phase space, these are found along horizontal lines. The other kind of waves are $g$-waves, which according to (2.3) have constant flow value, $f=$ const. Hence, it is useful to draw the level curves of $f$ in the $(s, g)$ diagram. This is done in figure 2.1. The bold curve labelled $T$ in the figure is the transition curve, where $f_{s}=0$. Solving the Riemann problem now consists of finding a sequence of $s$ - and $g$-waves that goes from $u_{L}$ to $u_{R}$. The solution to the Riemann problem may now be found in the $(s, g)$ phase space by the procedure indicated in figures 2.2 and 2.3 (use figure 2.2 for $u_{L}$ to the left of $T$, and figure 2.3 for $u_{L}$ to the right of $T$ ). Follow arrows that continuously connect $u_{L}$ to $u_{R}$. Then find the solution by graphing the corresponding waves in the $(x, t)$-plane in the direction of the arrows. Note that any Riemann problem gives a solution consisting of at most three waves, an $s$-wave, a $g$-wave, and another $s$-wave. We write this composite solution wave as $\left[u_{L} u_{R}\right]=s g s^{\prime}$. We close this section by displaying an example of how a Riemann problem like (2.2) is solved by the method indicated above. Given a Riemann problem as indicated in figure 2.4, in the $(s, f)$ plane the solution looks like figure 2.5: Starting with a shock moving backwards along $f_{l}$ from $s_{l}$ ( $s$-wave), crossing from $s_{-}$over to $s_{+}$at $f_{r}$ (minimal jump, $g$-wave), and finally continuing from $s_{+}$to $s_{r}$ with a rarefaction along $f_{r}$ (another $s$ - wave). In figure 2.6 we have indicated this solution in the $(s, g)$ phase space, and finally in figure 2.7 the solution in the $(x, t)$ plane is shown.

The front tracking scheme. In this section we present the scheme we will use to generate a sequence of approximate solutions to (2.1). This scheme is a generalization of Dafermos' [1] scheme for the scalar conservation law. The basic idea of this scheme is to generate a sequence of exact solutions to approximate equations obtained by taking a piecewise linear approximation of the flux function. Via a standard compactness argument we then show that this sequence posesses a convergent subsequence and furthermore that this converges towards a weak solution of (2.1). 
In order to define our approximation we first have to define the approximate flux functions. Roughly speaking these will be defined for a fixed $g$ to be piecewise linear continuous in $s$. Assume that $g_{0}(x)$ is a function taking values in the interval $[0, G]$. We make a partition of this interval by choosing equally spaced points $\left\{\tilde{g}_{i}\right\}_{1}^{\tilde{n}}$ such that $\frac{1}{\tilde{n}}=\delta$ for some fixed $\delta$. Let

$$
s_{T}(g)=\min _{s} f(s, g)
$$

and define $\tilde{f}_{i}=f\left(s_{T}\left(\tilde{g}_{i}\right), \tilde{g}_{i}\right)$. We choose $\left\{\hat{f}_{i}\right\}_{1}^{\hat{m}}$ to be an equally spaced partition of the interval $\left[f\left(s_{T}(G), G\right), 1\right]$ such that $\hat{m}=\left\lceil\frac{1}{\delta}\right\rceil+1$, where $\lceil x\rceil=$ the largest integer smaller than or equal to $x$. Let

$$
g_{T}(c)=\min _{f(s, g)=c} g
$$

and define $\hat{g}_{i}=g_{T}\left(\hat{f}_{i}\right)$. Now we define

$$
\left\{g_{j}\right\}_{1}^{N}=\left\{\tilde{g}_{j}\right\} \cup\left\{\hat{g}_{j}\right\} \quad\left\{f_{j}\right\}_{1}^{M}=\left\{\tilde{f}_{j}\right\} \cup\left\{\hat{f}_{j}\right\} .
$$

This defines a "mesh" in the $(s, g)$ plane (cf. figure 3.1) and we see that the solution to a Riemann problem defined by two points in the mesh will have intermediate states that are in the mesh. We wish to simplify the solution to the Riemann problem further still by requiring that the $s$-waves consist only of states that are part of the mesh. This we do by approximating $f\left(s, g_{i}\right)$ by a piecewise linear function for each $g_{i}$. More precisely let

$$
s_{0}(g)=0 \quad \text { and } \quad s_{i+1}(g)=\min \left\{s>s_{i}(g) \mid f(s, g) \in\left\{f_{i}\right\}_{1}^{M}\right\}
$$

for $i=1, \ldots, n(g)$, note that $s_{n(g)}(g)=1$. Let $s_{i, j}$ denote $s_{i}\left(g_{j}\right)$ and $f_{i, j}$ denote $f\left(s_{i, j}, g_{j}\right)$. We have that the intersections in the mesh have coordinates $\left(s_{i, j}, g_{j}\right)$, cf. figure 3.1. Finally we can define the approximate flux functions

$$
\begin{aligned}
& h_{\delta}\left(s, g_{j}\right)=f_{i, j}+s \frac{f_{i+1, j}-f_{i, j}}{s_{i+1, j}-s_{i, j}} \\
& f_{\delta}\left(s, g_{j}\right)=\left(h_{\delta}\left(s, g_{j}\right), 0\right)
\end{aligned}
$$

for $s \in\left[s_{i, j}, s_{i+1, j}\right]$ and for $j=1, \ldots, N$.

The solution of the Riemann problem defined by

$$
\begin{gathered}
s_{t}+h_{\delta}\left(s, g_{j}\right)_{x}=0 \\
s_{0}(x)= \begin{cases}s_{L} & \text { if } x<0 \\
s_{R} & \text { if } x \geq 0\end{cases}
\end{gathered}
$$

consists of a number of constant states separated by discontinuities moving apart. Furthermore these constant states are a subset of $\left\{s_{i, j}\right\}_{i=1}^{n\left(g_{j}\right)}$. For a complete discussion of the Riemann problem for piecewise linear flux functions see e.g. [4]. 
In the following we let $u$ denote the pair $(s, g)$ and $u_{i, j}$ denote $\left(s_{i, j}, g_{j}\right)$. Assume $u_{0}(x)$ to be a function taking values in the rectangle $[0,1] \times[0, G]$. We can construct an approximation to $u_{0}$, which we call $u_{0, \delta}(x)$, such that for each $x ; u_{0, \delta}(x) \in\left\{u_{i, j}\right\}$ and

$$
\lim _{\delta \rightarrow 0}\left\|u_{0}-u_{0, \delta}\right\|_{L_{1}}=0 \text {. }
$$

Condition (3.7) can be achived since $g_{j}-g_{j-1} \leq \delta$ and $s_{i, j}-s_{i-1, j}=O(\sqrt{\delta})$ where the righthand side of the last equation depends on $\frac{\partial^{2} f}{\partial s^{2}}$ on the $T$-curve.

We will now generate a weak solution $u_{\delta}(x, t)$ to the initial value problem

$$
u_{\delta t}+f_{\delta}\left(u_{\delta}\right)_{x}=0 \quad u_{\delta}(x, 0)=u_{0, \delta}(x) .
$$

The initial function $u_{0, \delta}$ defines a series of finitely many Riemann problems, and by construction the solution to these problems are constant states (which are included in the set $\left\{u_{i, j}\right\}$ ) separated by discontinuities. We can track these discontinuites and thereby propagate the solution forward in time, until two of them collide. At this point we have a situation similar to what we had initially, namely a sequence of Riemann problems. Therefore we can solve these and propagate the solution until the next collision. Note that by construction $u_{\delta}$ is a weak solution of (3.8). This process we call front tracking, and it is clear that it can be repeated an arbitrary number of times. We do however need to justify that we can propagate the solution in this manner up to any given time by a finite number of operations. But in order to do this we first define a certain functional $F$ which is nonincreasing for each collision of fronts.

We may think of a wave of $u_{\delta}$ either as a discontinuity in the $(x, t)$ plane or as a directed path in $(s, g)$ space. If the wave is an $s$-wave this path is just the straight line from the state to the left of the discontinuity to the state to the right of the discontinuity. If the wave is a $g$-wave the path is the curve $f=$ const. from the left state to the right state. Thus $u_{\delta}$ can be thought of as a finite sequence of connected waves in the $(s, g)$ plane representing the discontinuities in $u_{\delta}$ as we move from left to right in the $(x, t)$ plane. We will call any finite sequence of connected $s$ - or $g$-waves in the $(s, g)$ plane an $I$ curve, where by connected we mean that the left state of a wave in the sequence is the right state of its predessessor, and we say that an $I$ curve connects $u_{L}$ to $u_{R}$ if the left state of the first wave is $u_{L}$ and the right state of the final wave is $u_{R}$. We will use the techniques developed for the oil-polymer system by Temple[11] and construct a certain 1-1 mapping $\Psi$ from $(s, g)$ to $(z, g)$, and a functional $F(I)$ such that $F\left(u_{\delta}\right)$ dominates the total variation of $\Psi \circ u_{\delta}$. We then prove that $F\left(u_{\delta}\right)$ is nonincreasing for each collision, and that this implies firstly that the approximation procedure can be continued to any time by finitely many operations, and secondly that a subsequence of the approximate solutions converges in $L_{1}$.

The mapping $\Psi$ is similar to the mapping used by Temple in [11], and it involves the intersections of the $T$-curve with the level sets of $f$. Since $f$ does not take all values on $T$, we must extend both $T$ and the level sets outside $[0,1] \times[0, G]$. Assume for the moment that this is done in such a manner that for each point $(s, g)$ in $[0,1] \times[0, G]$ we can find a unique point $\left(s^{\prime}, g^{\prime}\right)$ on $T$ such that $f\left(s^{\prime}, g^{\prime}\right)=f(s, g)$. Then $z$ is defined as follows

$$
|z|=\left|g-g^{\prime}\right|
$$

$$
\text { sign } z= \begin{cases}-1 & \text { if }(s, g) \text { is to the left of } T \text { or above } T \\ 1 & \text { if }(s, g) \text { is to the right of } T \text { or below } T .\end{cases}
$$


We have two cases of how to define the point $\left(s^{\prime}, g^{\prime}\right)$ when $f$ does not take the value $f(s, g)$ on $T$, depending on whether $T$ intersects the $s$-axis or the $g$-axis. Assume first that $T$ intersects the $s$-axis. Then we can extend $T$ and the level curves of $f$ in a smooth manner such that they intersect $T$ at their minimum, cf. figure 3.2 If $T$ intersects the $g$ axis we make a smooth decreasing extension $g^{\prime}(s)$ of $T$ defined for negative $s$. If $f$ does not take the value $f(s, g)$ on $T$, then $f$ will take this value on the line $g=0$ at some point $\tilde{s}$. We then define $\left(s^{\prime}, g^{\prime}\right)=\left(-\tilde{s}, g^{\prime}(-\tilde{s})\right)$. Since the line $s=0$ is a level set for $f$ this mapping will be continuous and smooth, cf. figure 3.3. As in [11] we have that $\Psi$ is $1-1$ and regular everywhere exept on $T$. In the following we let $w=\Psi(u)$.

Now we can define the functional $F$. We define the strength of an $s$-wave to be

$$
|s|=|\Delta z|
$$

and and the strength of a $g$-wave

$$
|g|= \begin{cases}2|\Delta g| & \left\{\begin{array}{l}
\text { if } g \text { is to the right of } T \text { and } g_{L}<g_{R} \\
\text { or } g \text { is to the left of } T \text { and } g_{L}>g_{R}
\end{array}\right. \\
4|\Delta g| & \left\{\begin{array}{l}
\text { if } g \text { is to the right of } T \text { and } g_{L}>g_{R} \\
\text { or } g \text { is to the left of } T \text { and } g_{L}<g_{R} .
\end{array}\right.\end{cases}
$$

We can write $u_{\delta}$ as $b_{1}, \ldots, b_{n}=I$, where $b_{i}$ is either an $s$-wave or a $g$-wave and we define

$$
F(I)=\sum_{i}\left|b_{i}\right|
$$

Here follows the main lemma regarding $F$.

LEMMA 3.1. Let $J$ be any $I$ curve connecting $u_{L}$ to $u_{R}$, and let $\left[u_{L} u_{R}\right]$ be the $I$ curve that solves the Riemann problem defined by $u_{L}$ and $u_{R}$. Then $F\left(\left[u_{L} u_{R}\right]\right) \leq F(J)$.

The proof of this lemma is analogous to the proof of the corresponding lemma (Lemma 5.1 ) in [11] and since it involves the study of a number of cases, it is presented in an appendix.

Now let $F_{0}$ denote $F\left(u_{\delta}(\cdot, t)\right)$ where $t$ is taken to be so small that no collision has yet occured. The main theorem of this section then follows immediately from Lemma 3.1.

ThEOREM 3.1. Assume that $F_{0}$ is finite, and let $t_{1} \leq t_{2}$, then

$$
F\left(u_{\delta}\left(\cdot, t_{1}\right)\right) \geq F\left(u_{\delta}\left(\cdot, t_{2}\right)\right)
$$

Proof: It is clear that $F$ only changes value when we have a collision of discontinuities in $u_{\delta}$. At any one time we can have only finitely many collisions, and the change in $F$ is a sum of the changes in $F$ at each collision point. Consider therefore two discontinuities that collide, the one on the left separating states $u_{L}$ and $u_{M}$, the one on the right separating states $u_{M}$ and $u_{R}$. The theorem now follows since by lemma $3.1 F\left(\left[u_{L} u_{M}\right]\left[u_{M} u_{R}\right]\right) \geq$ $F\left(\left[u_{L} u_{R}\right]\right)$. 
It is clear that $F$ at each collision of $u_{\delta}$ either remains constant or changes by at least $\Delta$, where $\Delta$ is the minimum distance between the states of which $u_{\delta}$ may consist, i.e.

$$
\Delta=\min _{(i, j) \neq(k, l)}\left|w_{i, j}-w_{k, l}\right|
$$

We wish to investigate those collisions which are possible if $F$ remains constant for all time. Let $s^{+}\left(s^{-}\right)$denote those $s$-waves over which $s$ is increasing (decreasing), and let $s_{R}$ $\left(s_{L}\right)$ denote an $s$-wave with left and right state to the right (left) of $T$.

LEMMA 3.2. Assume that $F\left(u_{\delta}\right)$ is constant and that $u_{\delta}$ contains the wave sequence $g s_{R}^{+}$ $\left(s_{L} g\right)$. Then no $s$-wave will collide from the right (left) with $g$.

Proof: Let the $s_{R}^{+}$wave separate states $s_{l}<s_{r}$. Since $F$ is constant it can only collide with $s$-waves that separate states $s_{r}<s^{\prime}$. The result of this collision is a single $s_{R}^{+}$wave separating states $s_{l}<s^{\prime}$. If the $s$-wave collides with a $g$-wave the result of the collision must be $g s_{R}^{+}$since $F$ is constant. Since all $s_{R}$ waves have positive speeds the lemma follows. An analogous argument takes care of the case $s_{L} g$.

LEMmA 3.3. Assume that $F\left(u_{\delta}\right)$ is constant, then only three types of collisions can occur:

(1) An $s$-wave separating states $s_{l} \lessgtr s_{m}$ colliding with another $s$-wave separating states $s_{m} \lessgtr s_{r}$, giving as a result a single $s$-wave separating $s_{l} \lessgtr s_{r}$.

(2) An $s$-wave colliding with a $g$-wave from the right (left), giving $s g$ ( $g s$ ) as result ('an $s$-wave passing through a $g$-wave').

(3) An $s$-wave colliding with a $g$-wave, giving $s_{L}^{-} g s_{R}^{+}$as a result.

The proof of this lemma consists of checking a number of cases of Riemann solutions in the diagrams 2.2 and 2.3. It is straightforward and is therefore omitted. Combining the last two lemmas we see that if $F$ is constant, our approximation $u_{\delta}$ is well defined. However Theorem 3.1 implies that after some finite number of collisions, $F$ will change by an amount less than $\Delta$ for all subsequent collisions, i.e. $F$ will remain constant for all collisions thereafter. Therefore the approximation $u_{\delta}$ is well defined and $u_{\delta}$ is constant on a finite number of regions in $\mathbb{R} \times \mathbb{R}^{+}$. These regions are separated by a finite number of straight lines.

Convergence of solution. Let $\operatorname{Var}_{a b} u$ denote the total variation of $u$ with respect to the variables $a$ and $b$. By construction, $F(I) \geq \operatorname{Var}_{z g} J$ for any $I$-curve $J$. Hence, by Theorem 3.1 we may find a uniform bound on $\operatorname{Var}_{z g} u_{\delta}(\cdot, t)$, provided $F_{0}$ is bounded. We show this by applying Temple's argument [11]: Let $S(\epsilon)$ be a strip of width $\epsilon$ around the $T$-curve. If $u_{L}$ or $u_{R}$ are outside the strip, the Riemann problem solution $\left[u_{L} u_{R}\right]$ has a finite number of waves, each globally bounded, and the waves intersect transversally in the $(s, g)$ plane. Thus, $\operatorname{Var}_{z g}\left[u_{L} u_{R}\right]=O(1)\left|w_{R}-w_{L}\right|$ if $w_{R}=\left(z_{R}, g_{R}\right)$ and $w_{L}=\left(z_{L}, g_{L}\right)$ are the images of $u_{R}$ and $u_{L}$ under $\Psi$. Secondly, if $u_{L}$ and $u_{R}$ are in $S(\epsilon), \operatorname{Var}_{z g}<5\left|w_{L}-w_{R}\right|$ by construction, for $\epsilon$ sufficiently small. Therefore, for any Riemann problem, $\operatorname{Var}_{z g} I=O(1)\left|w_{L}-w_{R}\right|$. 
Let $\left\{J_{i}\right\}$ denote the solutions of the initial Riemann problems. This gives

$$
\begin{aligned}
F_{0} & \leq 4 \sum_{i} \operatorname{Var}_{z g} J_{i} \\
& \leq O(1) \sum\left|w_{L}-w_{R}\right| \\
& \leq O(1) \operatorname{Var}_{z g} u_{0, \delta}
\end{aligned}
$$

proving that $\operatorname{Var}_{z g} u_{\delta}(\cdot, t)$ is bounded for each fixed $t$.

Having proved boundedness in space for each time $t$, we want to prove Lipschitz continuity in $t$ :

LEMMA 4.1.

$$
\int_{-\infty}^{\infty}\left|w_{\delta}\left(x, t_{2}\right)-w_{\delta}\left(x, t_{1}\right)\right| d x=O(1)\left|t_{2}-t_{1}\right| \operatorname{Var}_{z g} u_{0, \delta}
$$

Proof: Let $M$ be the maximum speed at which a wave may propagate. $M$ is given by the maximum slope of any $f_{\delta}\left(\cdot, g_{j}\right)$. Thus, if $t_{1}<t_{2},\left|w_{\delta}\left(x, t_{2}\right)-w_{\delta}\left(x, t_{1}\right)\right|$ is bounded by the spatial variation of $w_{\delta}\left(y, t_{1}\right)$, where $x-M\left|t_{2}-t_{1}\right|<y<x+M\left|t_{2}-t_{1}\right|$. However, as pointed out above, $w_{\delta}(\cdot, t)$ is of bounded variation, so that we may write:

$$
\int_{-\infty}^{\infty}\left|w_{\delta}\left(x, t_{2}\right)-w_{\delta}\left(x, t_{1}\right)\right| d x=O(1) \cdot \int_{-\infty}^{\infty} \int_{x-M\left|t_{2}-t_{1}\right|}^{x+M\left|t_{2}-t_{1}\right|}\left|\frac{d w_{\delta}}{d y}\right| d x d y
$$

Here, $\left|\frac{d w_{\delta}}{d y}\right| d x d y$ is a measure of mass $\operatorname{Var}_{z g} w_{\delta}(x, t)$, and by changing the order of integration we have:

$$
\begin{aligned}
& \int_{-\infty}^{\infty}\left|w_{\delta}\left(x, t_{2}\right)-w_{\delta}\left(x, t_{1}\right)\right| d x= \\
& \left.\left.O(1) M \mid t_{2}-t_{1}\right] \operatorname{Var}_{z g} w_{\delta}(x, t)=O(1) M \mid t_{2}-t_{1}\right] \operatorname{Var}_{z g} u_{0, \delta}(x)
\end{aligned}
$$

the last equality follows from Lemma 4.1 .

It remains to prove the convergence of the sequence $\left\{u_{\delta}\right\}$.

THEOREM 4.1. Let $w_{0}=\Psi u_{0}$ be any initial data such that $\operatorname{Var}_{z g} w_{0}<\infty$. Then for any sequence $\{\delta\}$, such that $\delta \rightarrow 0$, there exists a subsequence $\delta_{j}$ and a function $u$, such that for any finite time $T, u_{\delta_{j}}(\cdot, t)$ converges uniformly to $u(\cdot, t)$ in $L_{\text {loc }}^{1}(x)$ for any $t \leq T$.

Proof: We have demonstrated that $w_{\delta}(\cdot, t)$ has uniformly bounded variation for each $t$, and so it follows from Helly's theorem, that a subsequence converges in $L_{\text {loc }}^{1}(x)$. By a diagonalization argument, such convergence is achieved on a countable, dense set of rational $t$-values, $t_{h, k}=h / k, 0 \leq t_{h, k} \leq T$. Let $w_{\delta(h, k)}$ be this subsequence. By Lemma 4, we may find a subsequence $w_{\delta(l)}$ that converges uniformly in $L^{1}[-M, M]$ at fixed $t$. Thus for this sequence:

$$
\begin{aligned}
& \int_{-M}^{M}\left|w_{i}(x, t)-w_{j}(x, t)\right| d x \leq \\
& \int_{-M}^{M}\left|w_{i}(x, t)-w_{i}\left(x, t_{h, k}\right)\right| d x+\int_{-M}^{M}\left|w_{i}\left(x, t_{h, k}\right)-w_{j}\left(x, t_{h, k}\right)\right| d x+ \\
& \quad \int_{-M}^{M}\left|w_{j}\left(x, t_{h, k}\right)-w_{j}(x, t)\right| d x
\end{aligned}
$$


Here the first and third term approach zero by Lemma 4.1, and the second term is small by the boundedness of $w_{\delta}(\cdot, t)$. Therefore $w_{\delta(l)}$ converges uniformly in $L^{1}[-M, M]$. This argument may now be applied a countable number of times, concluding the existence of a sequence $\left\{w_{\delta_{j}}\right\}$ such that for any $t>0, w_{\delta_{j}}(\cdot, t) \rightarrow w(\cdot, t)$ uniformly. The uniform continuity of $\Psi^{-1}$ gives the theorem for $u_{\delta_{j}}=\Psi^{-1} w_{\delta_{j}}$ and $u=\Psi^{-1} w$.

Finally, we want to show that the limit obtained above is indeed a weak solution to the problem (2.1). For $T<\infty$ we define:

$$
W(u)=\int_{-\infty}^{\infty} \int_{0}^{T}\left(\phi_{t} u+\phi_{x} f(u)\right) d x d t+\int_{-\infty}^{\infty} \phi u_{o} d x
$$

for $\phi=\phi(x, t)$ an arbitrary function in $C_{0}^{1}$. To simplify the notation we let $\delta$ denote a member of the convergent subsequence $\left\{\delta_{j}\right\}$. For $u=\lim _{\delta \rightarrow 0} u_{\delta}$ we want to show that $W(u)=0$. Since $u_{\delta}$ is a weak solution of $(3.8)$ :

$$
\int_{-\infty}^{\infty} \int_{0}^{T}\left(\phi_{t} u_{\delta}+\phi_{x} f_{\delta}\left(u_{\delta}\right)\right) d x d t+\int_{-\infty}^{\infty} \phi u_{0, \delta} d x=0
$$

which gives:

$$
W(u)=\int_{-\infty}^{\infty} \int_{0}^{T}\left(\phi_{t}\left(u-u_{\delta}\right)+\phi_{x}\left(f(u)-f_{\delta}\left(u_{\delta}\right)\right)\right) d x d t+\int_{-\infty}^{\infty} \phi\left(u_{0}-u_{0, \delta}\right) d x
$$

for all $\delta$. Thus

$$
\begin{aligned}
|W(u)| \leq & \left\|\phi_{t}\right\|_{\infty}\left\|u-u_{\delta}\right\|_{L^{1}}+\left\|\phi_{x}\right\|_{\infty}\left\|f(u)-f_{\delta}\left(u_{\delta}\right)\right\|_{L^{1}}+\|\phi\|_{\infty}\left\|u_{0}-u_{0, \delta}\right\|_{L^{1}} \\
\leq & \left\|\phi_{t}\right\|_{\infty}\left\|u-u_{\delta}\right\|_{L^{1}} \\
& +\left\|\phi_{x}\right\|_{\infty}\left\|f(u)-f_{\delta}(u)\right\|_{L^{1}} \\
& +\left\|\phi_{x}\right\|_{\infty}\left\|f_{\delta}(u)-f_{\delta}\left(u_{\delta}\right)\right\|_{L^{1}} \\
& +\|\phi\|_{\infty}\left\|u_{0}-u_{0, \delta}\right\|_{L^{1}}
\end{aligned}
$$

Here, by Lipschitz continuity of $f$ and $f_{\delta}$, the terms (4.2b) and (4.2c) above are small. Furthermore (4.2a) is small by the construction of $u$ as the $L^{1}$ limit of $u_{\delta}$, and (4.2d) is small by the construction of $u_{0, \delta}$. Hence, for any given $\epsilon>0$, we may choose $\delta$ so that $|W(u)|<\epsilon$, concluding that $W(u)=0$, and the limit $u$ is a weak solution of (2.1).

Remark. The system of conservation laws modelling polymer flow in porous media

$$
\begin{aligned}
& s_{t}+(g(s, b) s)_{x}=0 \\
& b_{t}+(g(s, b) b)_{x}=0
\end{aligned}
$$

studied by Temple[11], and Temple and Isaacson[7], [8], has a structure of the solution to the Riemann problem that is remarkably similar to the Riemann solution used in the present paper (Compare figures 8 and 9 in [11] with figures 2.2-2.3). It is this similarity 
that enabled us to use essentially the same techniques as [11] to show that the functional $F$ was nonincreasing and to obtain the estimates on $\operatorname{Var}_{z g} w_{\delta}$. This in turn guaranteed that our approximation $u_{\delta}$ was well defined and that the sequence $\left\{u_{\delta}\right\}$ posessed a subsequence which converged towards a weak solution of (2.1). We could have defined an analogue of $u_{\delta},\left(s_{\delta}, b_{\delta}\right)$ as an approximation to the solution of (4.3). Since the whole subsequent argument hinges on the fact that $F$ is nonincreasing, it applies equally well to $\left(s_{\delta}, b_{\delta}\right)$ as to $u_{\delta}$. Therefore, the front tracking method presented here gives an alternative proof of the existence of a solution to the Cauchy problem for (4.3).

Appendix. Here we present the proof of Lemma 3.1:

LEMMA 3.1. Let $J$ be any $I$ curve connecting $u_{L}$ to $u_{R}$, and let $\left[u_{L} u_{R}\right]$ be the $I$ curve that solves the Riemann problem defined by $u_{L}$ and $u_{R}$. Then $F\left(\left[u_{L} u_{R}\right]\right) \leq F(J)$.

Since the structure of the solution of the Riemann problem is similar to the structure of the solution of the Riemann problem for the polymer system studied by Temple[11], lemma 3.1 is proved by essentially the same arguments as the corresponding lemma in [11]. We first prove three Lemmas, lemma 3.1 will then follow from these.

Let $g^{+}\left(g^{-}\right)$denote a $g$ wave over which $s$ is increasing (decreasing), and let $g_{R}\left(g_{L}\right)$ denote a $g$-wave to the right (left) of $T$. We can now define the "addition" of waves; the addition of $s_{1}$ and $s_{2}$ is the $s$-wave that goes from the left state of $s_{1}$ to the right state of $s_{2}$. If $g_{1}$ and $g_{2}$ are both $g_{L}$ or $g_{R}$ waves then the addition of $g_{1}$ and $g_{2}$ is the combined $g$-wave. If two $g$-waves are of different type and $g\left(u_{L}\right)<g\left(u_{R}\right)\left(g\left(u_{L}\right)>g\left(u_{R}\right)\right)$ then their addition is the unique wave $g s(s g)$ that goes from $u_{L}$ to $u_{R}$.

Assume now that $J=s g(J=g s)$ connects $u_{L}$ to $u_{R}$. If $u_{L}$ and $u_{R}$ are on the same side of $T$ and a "parallelogram" of $s$ and $g$ waves can be drawn with $u_{L}$ and $u_{R}$ as diagonally opposed corners, the interchange of $J$; $\bar{J}$, is defined to be the unique $I$ curve $\bar{g} \bar{s}(\bar{s} \bar{g})$ that connects $u_{L}$ to $u_{R}$. If $u_{L}$ and $u_{R}$ are on different sides of $T$ we can only define $\bar{J}$ if $J=s g_{L}^{-}$ or $J=g_{R}^{+} s$. The interchange of $s g_{L}^{-}$is the unique $I$ curve $g_{R}^{+} \bar{s}$ that connects the same endpoints. Other $I$-curves do not have an interchange. As in [11] it is easy to show that if $J$ is the addition of $b_{1}$ and $b_{2}$ then $F\left(b_{1} b_{2}\right) \geq F(J)$, and if $\bar{J}$ is the interchange of $J$ then $F(J)=F(\bar{J})$. Furthermore if $J$ connects $u_{L}$ to $u_{R}$ and $J$ has an interchange $\bar{J}$, then $\left[u_{L} u_{R}\right]=J$ or $\left[u_{L} u_{R}\right]=\bar{J}$.

Lemma A1. If $J$ connects $u_{L}$ to $u_{R}$ and $J=g s$ or $J=s g$ then $F(J) \geq F\left(\left[u_{L} u_{R}\right]\right)$.

PROOF: If $J$ has an interchange then the lemma holds. Assuming that $J$ does not have an interchange we have eight cases to check: $J=s g$ or $g s, g=g_{R}$ or $g_{L}, g=g^{+}$or $g^{-}$. But if $J=g_{R}^{+} s$ or $s g_{L}^{-}$then $J$ has an interchange. This leaves six cases which are checked in figure $\mathrm{A} 1$.

LemmA A2. If $J$ connects $u_{L}$ to $u_{R}$ and $J=\operatorname{sgs} s^{\prime}$ then $F(J) \geq F\left(\left[u_{L} u_{R}\right]\right)$.

PROOF: If $g s$ or $s g^{\prime}$ can be interchanged, we can interchange and add waves so that lemma A1 applies. Assume therefore that neither can be interchanged. This implies that $s$ and $s^{\prime}$ both cross $T$ and that $g=g_{L}^{+}$or $g_{R}^{-}$. This leaves two cases to check as an exercise for the reader. 
LEMMA A3. If $J$ connects $u_{L}$ to $u_{R}$ and $J=g s g^{\prime}$ then $F(J) \geq F\left(\left[u_{L} u_{R}\right]\right)$.

Proof: We can assume that $s$ crosses $T$ and that $g \neq g_{R}^{+}$and $g^{\prime} \neq g_{L}$. Also if the variable $g$ is increasing over $g$ and decreasing over $g^{\prime}$ (or vice versa) it is easy to show that $F(J) \geq F(\tilde{s} \tilde{g})$ or $F(\tilde{g} \tilde{s})$ where $\tilde{s} \tilde{g}$ or $\tilde{g} \tilde{s}$ connects $u_{L}$ to $u_{R}$. In this case we can now use lemma $A 1$. Now $s$ can cross from left to right or right to left, i.e. $g s g^{\prime}=g_{L}^{+} s g_{R}^{-}$or $g_{R}^{-} s g_{L}^{+}$. In both of these cases $J$ contains a "strong" $g$-wave, whereas $\left[u_{L} u_{R}\right]=s g^{+} s^{\prime}$, i.e. the Riemann solution has a "weak" $g$-wave. The presence of this strong wave makes the lemma hold.

Proof of Lemma 3.1: Once lemmas A2 and A3 are established, the proof of this lemma carries over literally from the proof of Lemma 5.1 in [11] if ' $c$-waves' are substituted with ' $g$-waves'.

\section{REFERENCES}

1. C.M. Dafermos, Polygonal approximation of solution to the initial value problem for a conservation law, J. Math. Anal. Appl. 38 (1972), 33-41.

2. T. Gimse, A numerical method for a system of equations modelling one-dimensional three-phase flow in a porous medium, Notes on Num. Fluid Mech. 24 (1989), 159-168.

3. T. Gimse, N.H. Risebro, Riemann problems with a discontinuous flux function, in "Proceedings from the Third International Conference on Hyperbolic Problems," Uppsala, 1990 (to appear).

4. H. Holden, L. Holden, R. Høegh-Krohn, A numerical method for first order nonlinear scalar conservation laws in one dimension, Comput. Math. Applic. 15 (1988), 595-602.

5. L. Holden, R. Høegh-Krohn, A class of $N$ nonlinear hyperbolic conservation laws, J. Diff. Eqns. 84 (1990), 73-99.

6. E. Isaacson, Global solution of a Riemann problem for a non-strictly hyperbolic system of conservation laws arising in enhanced oil recovery, J. Comput. Phys. (to appear).

7. E. Isaacson, B. Temple, Analysis of a singular system of conservation laws, J. Diff. Eqns. 65 (1986), 250-268.

8. E. Isaacson, B. Temple, Structure of asymptotic states in a singular system of conservation laws, Adv. Appl. Math. 11 (1990), 205-219.

9. N. Krushkov, Quasi-linear equations of the first order, Matem. Sbornik 2 (1970), 217-243.

10. D.W. Peaceman, "Fundamentals of numerical reservoir simulation," Elsevier, Amsterdam, 1977.

11. B. Temple, Global solution of the Cauchy problem for a class of $2 \times 2$ non-strictly hyperbolic conservation laws, Adv. Appl. Math. 3 (1982), 335-375. 


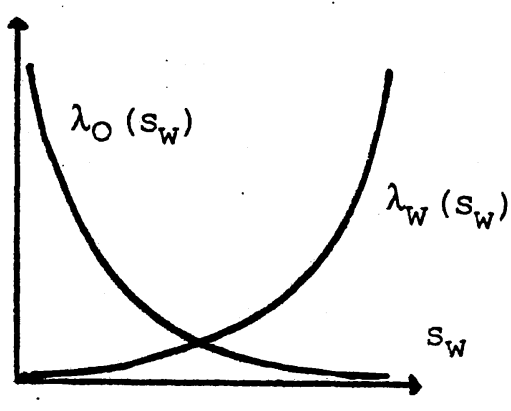

Figure 1.1

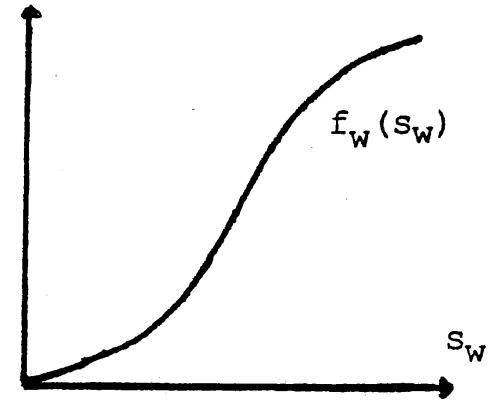

Figure 1.2

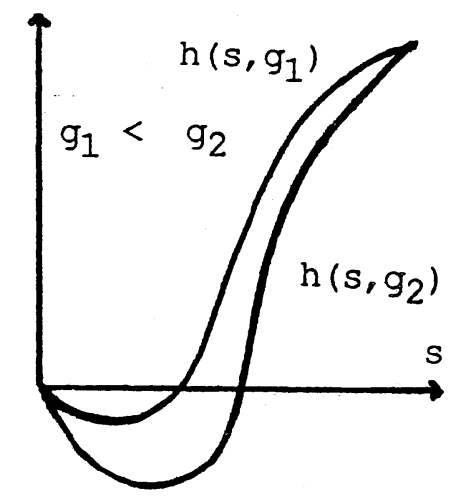

Figure 1.3

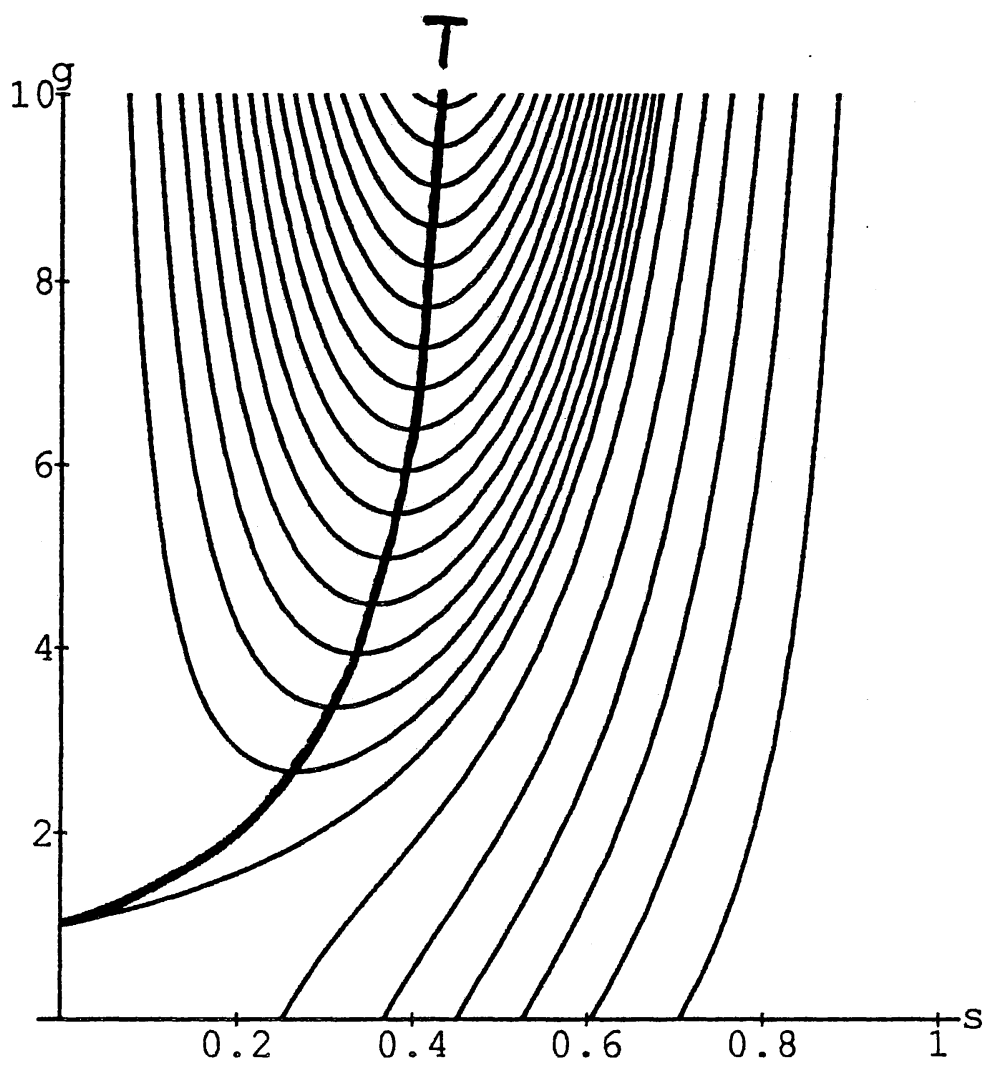

FIGURE 2.1 


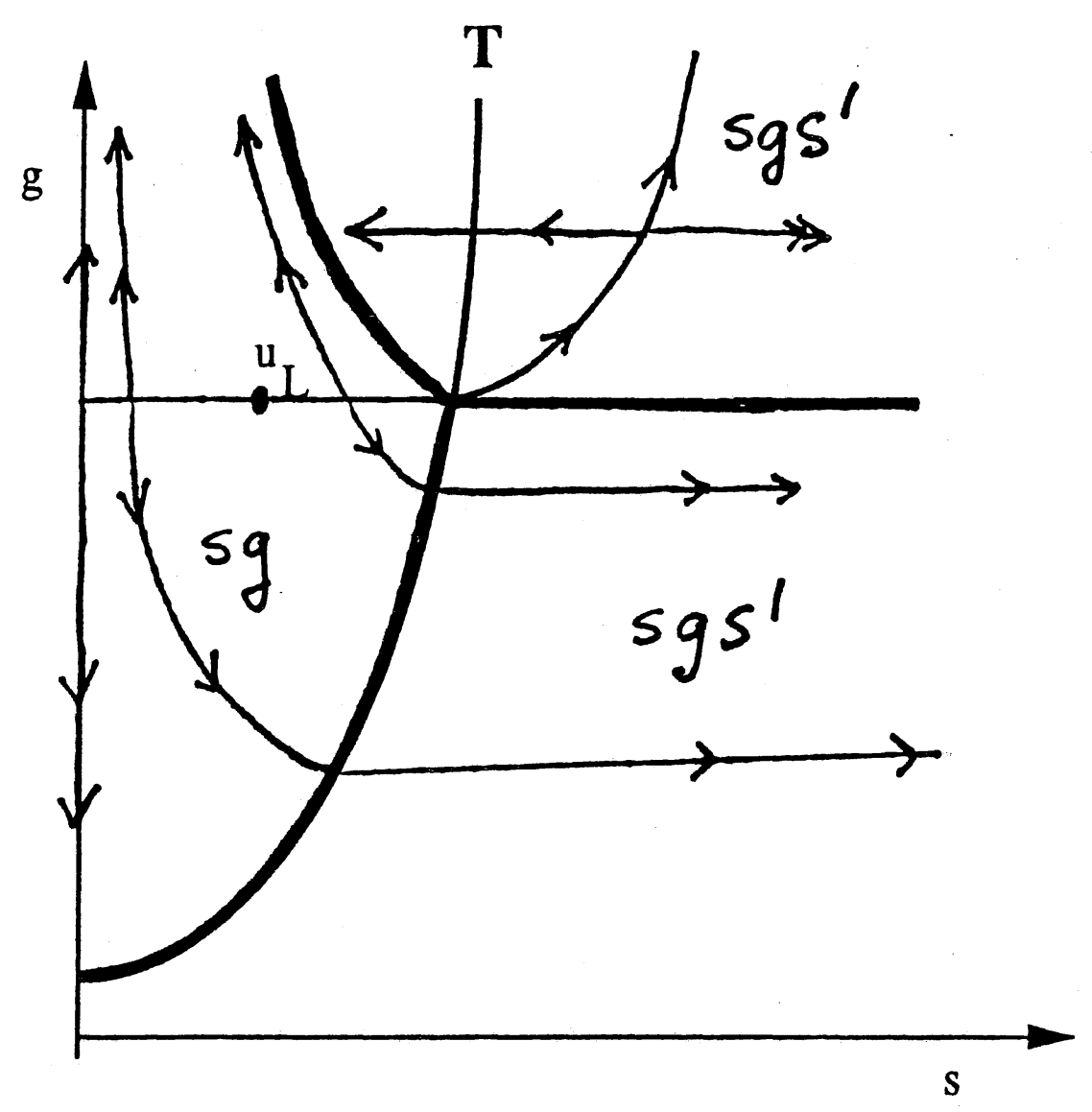

Figure 2.2

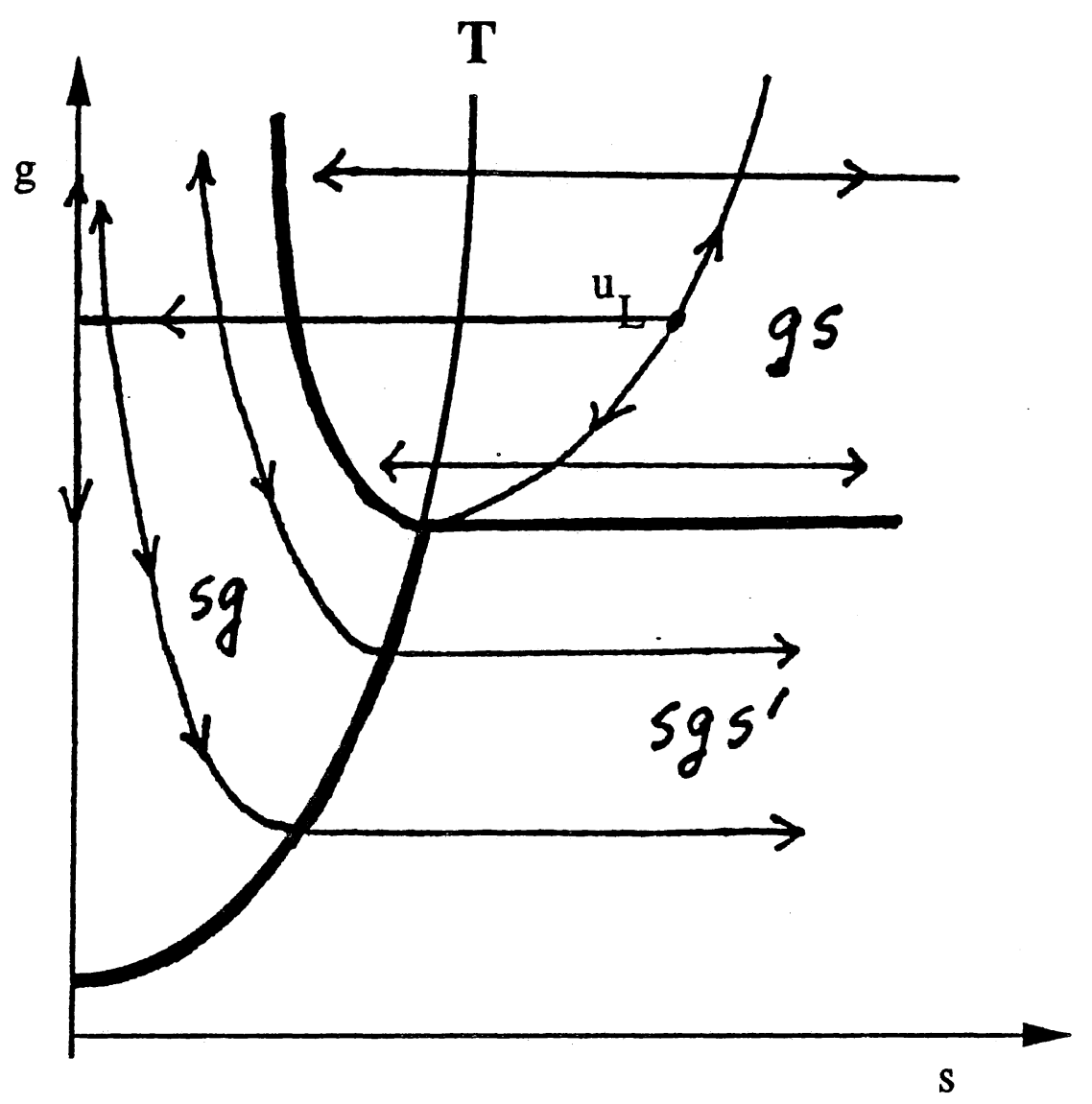

Figure 2.3 


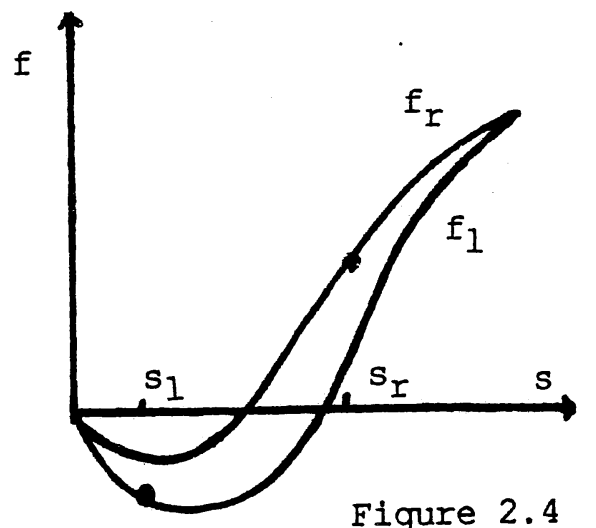

Figure 2.4

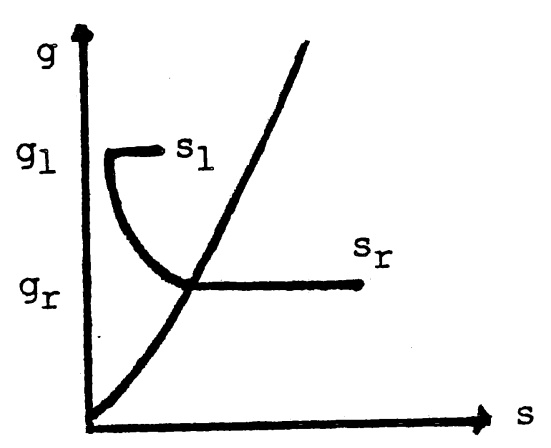

Figure 2.6
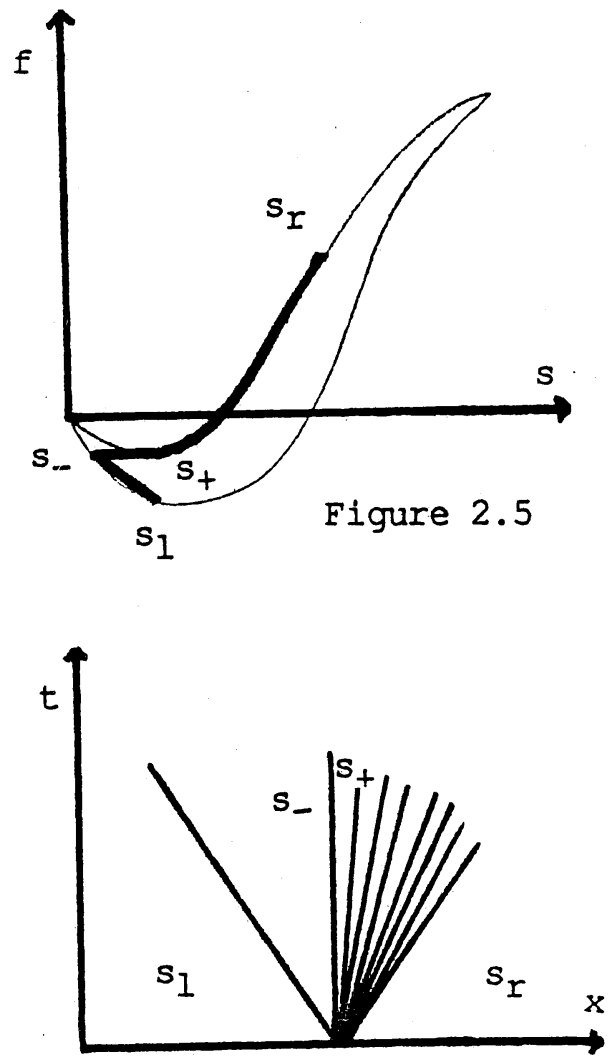

Figure 2.7

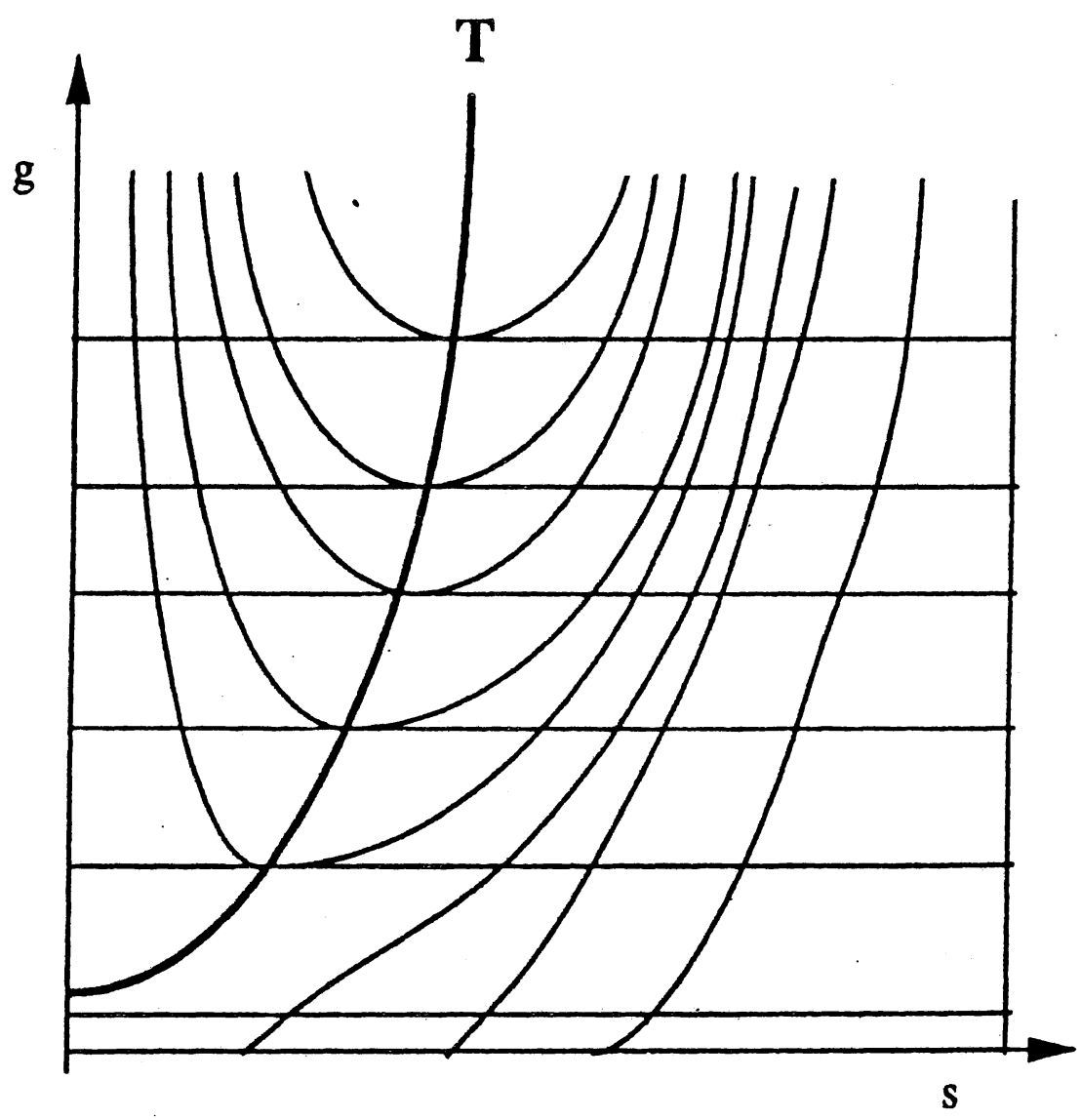

Figure 3.1 

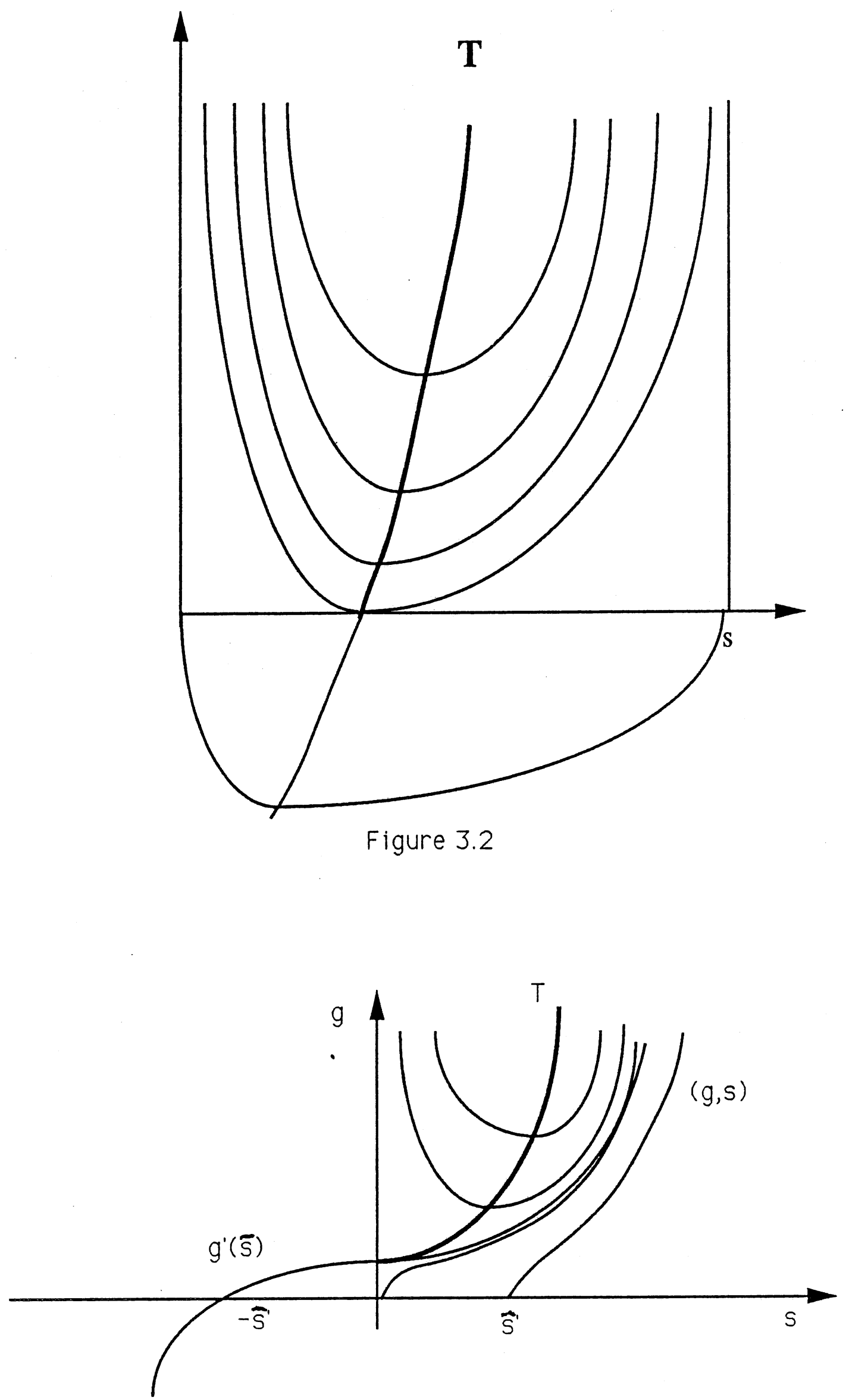

Figure 3.3 
$R+$

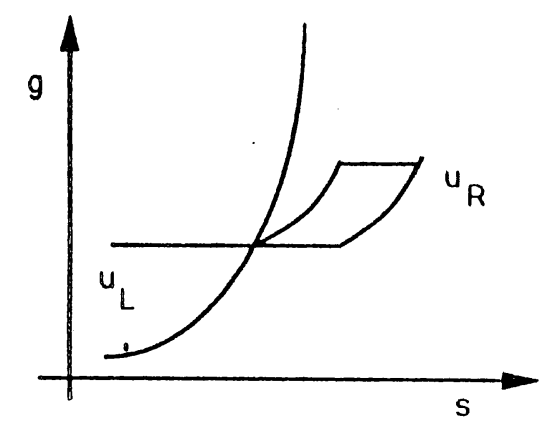

In this case $F(J)=F(1)$ as is

seen from the figure.
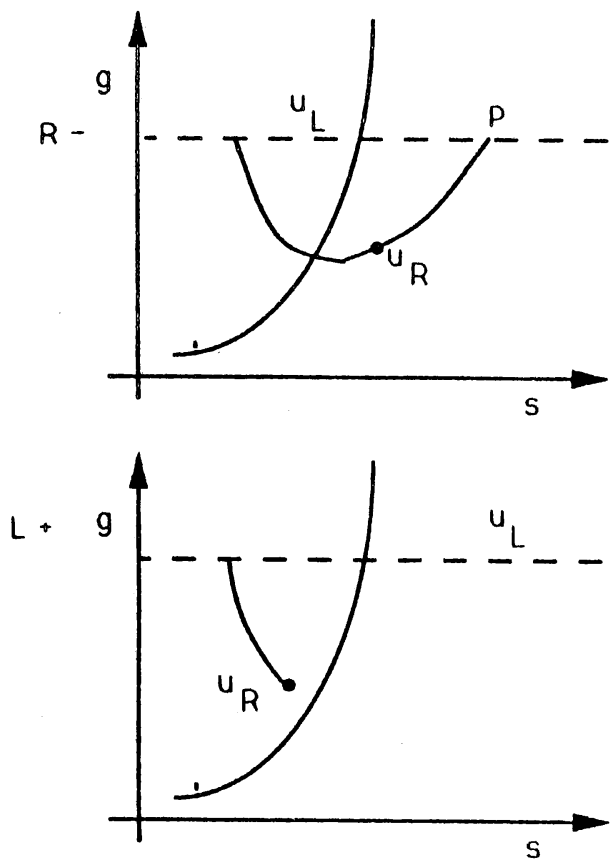

$J=g S$

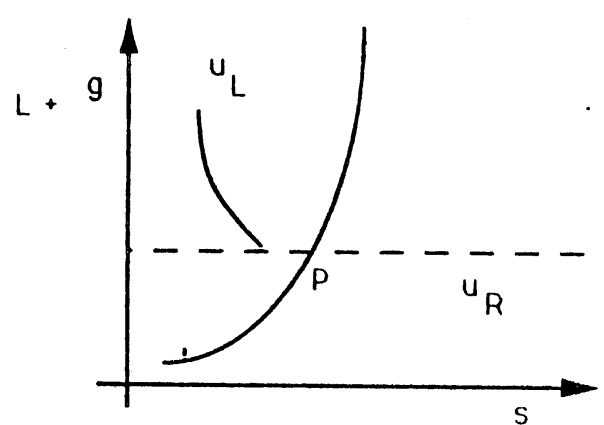

FIGURE Al

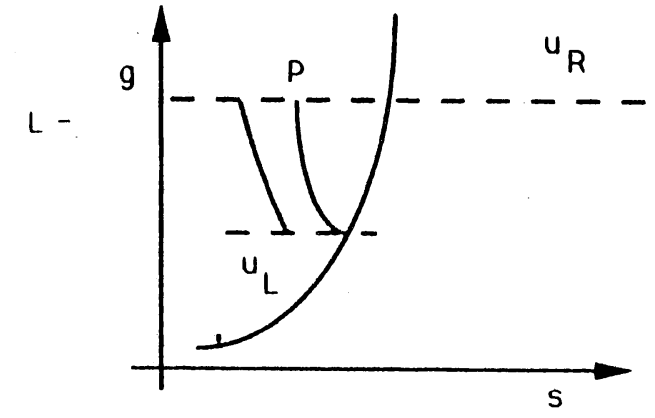

If $U_{R}$ is to the left of $P$ then we can interchange. Else the solution of the Riemann problem is $s g_{L}^{+} s$ and $F(1)<F(J)$

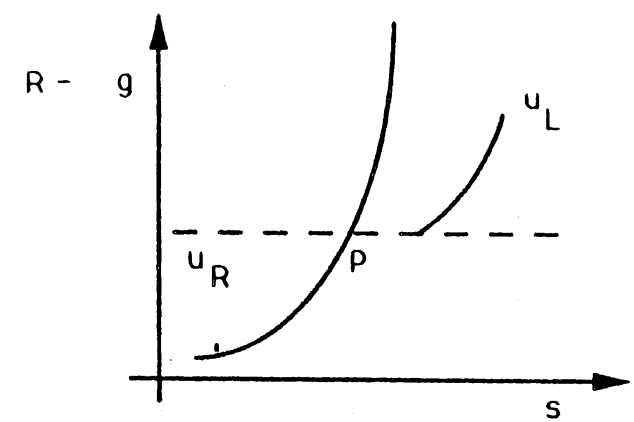

In this case the solution of the Riemann problem is either sg or gs and $F(1)=F(J)$ in both cases.
In this case the solution of the Riemann problem is either $\mathrm{sg}$ or gs. $F(1)=F(J)$ in both cases.

If $U_{R}$ is to the left of $P$ then we can interchange. Else the solution of the Riemann problem is sgs' and $F(I)=F(J)$
Figure Al (CONTINUED) 